\title{
Toward Improved Environmental Stability of Polymer:Fullerene and Polymer:Nonfullerene Organic Solar Cells: A Common Energetic Origin of Light- and Oxygen-Induced Degradation
}

Emily M. Speller, ${ }^{\dagger}$ Andrew J. Clarke, ${ }^{\dagger}$ Nicholas Aristidou, ${ }^{\ddagger}$ Mark F. Wyatt, ${ }^{\S}$ Laia Francàs, ${ }^{\dagger}$ George Fish, ${ }^{\ddagger}$ Hyojung Cha, ${ }^{\ddagger}$ Harrison Ka Hin Lee, ${ }^{\dagger}$ Joel Luke, ${ }^{\| \odot}$ Andrew Wadsworth, ${ }^{\ddagger \odot}$ Alex D. Evans, ${ }^{\#, \nabla^{8}}$

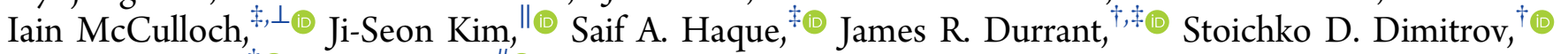
Wing C. Tsoi, $* \dagger \oplus$ and Zhe $\mathrm{Li}^{*}, \#($ )

${ }^{\dagger}$ SPECIFIC, College of Engineering, Swansea University, Bay Campus, Fabian Way, Swansea SA1 8EN, United Kingdom ${ }^{\ddagger}$ Department of Chemistry and Centre for Plastic Electronics, Imperial College London, London SW7 2AZ, United Kingdom ${ }^{\S}$ EPSRC UK National Mass Spectrometry Facility (NMSF), Swansea University Medical School, Wales SA2 8PP, United Kingdom "Department of Physics and Centre for Plastic Electronics, Imperial College London, London SW7 2AZ, United Kingdom

${ }^{\perp}$ Physical Sciences and Engineering Division, KAUST Solar Center (KSC), King Abdullah University of Science and Technology (KAUST), Thuwal 23955-6900, Saudi Arabia

${ }^{\#}$ School of Engineering, Cardiff University, Newport Road, Cardiff CF24 3AA, United Kingdom

${ }^{\nabla}$ School of Physics and Astronomy, Cardiff University, Newport Road, Cardiff CF24 3AA, United Kingdom

Supporting Information

ABSTRACT: With the emergence of nonfullerene electron acceptors resulting in further breakthroughs in the performance of organic solar cells, there is now an urgent need to understand their degradation mechanisms in order to improve their intrinsic stability through better material design. In this study, we present quantitative evidence for a common root cause of light-induced degradation of polymer:nonfullerene and polymer:fullerene organic solar cells in air, namely, a fast photo-oxidation process of the photoactive materials mediated by the formation of superoxide radical ions, whose yield is found to be strongly controlled by the lowest unoccupied molecular orbital (LUMO) levels of the electron acceptors used. Our results elucidate the general relevance of this degradation mechanism to both polymer:fullerene and polymer:nonfullerene blends and highlight the necessity of designing electron acceptor materials with sufficient electron affinities to overcome this challenge, thereby paving the way toward achieving long-term solar cell stability with minimal device encapsulation.

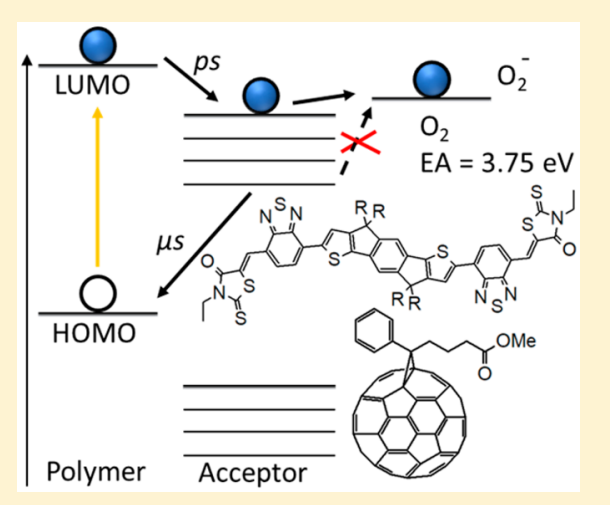

$\mathrm{T}$ $\checkmark$ he recent emergence of nonfullerene acceptors (NFAs) has reinvigorated the field of organic solar cells (OSCs), with their device efficiencies rocketing from $\sim 3$ to over $17 \%$ over the past $3-4$ years, ${ }^{1-3}$ already exceeding the threshold for commercial viability. In addition to potentially reduced material complexity and fabrication costs compared to fullerene derivatives, this class of electron acceptor materials further possess excellent optical and electrical properties owing to their highly tunable molecular structures. The synthetic flexibility of NFAs allows their optical bandgap to be tuned, allowing the light harvesting properties to be optimized in chosen spectral regions, e.g., NIR or UV. The opportunity to tune the molecular orbital energetics of NFAs also facilitates the engineering of devices with opencircuit voltages of over $1.1 \mathrm{eV}$ and a remarkably low voltage loss. ${ }^{4,5}$

Received: January 15, 2019

Accepted: March 12, 2019

Published: March 12, 2019 

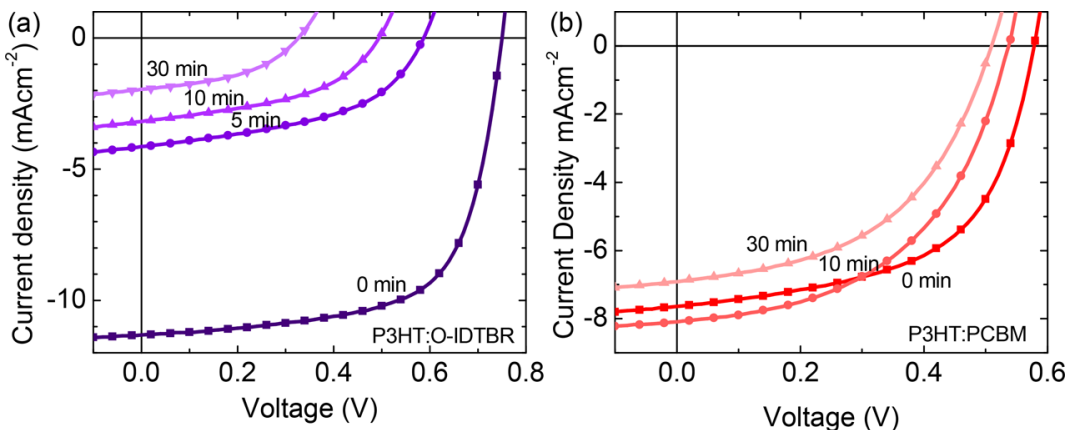

Figure 1. Current density-voltage $(J-V)$ characteristics of OSCs with a structure of (a) ITO/ZnO/P3HT:O-IDTBR/MoO $/ \mathrm{Ag}^{\mathrm{An}}$ and $(\mathrm{b})$ ITO/ZnO/P3HT:PCBM/ $/ \mathrm{MoO}_{3} / \mathrm{Ag}$, undergoing different photoaging times under simulated AM1.5G illumination in dry air prior to electrode deposition.

With the majority of research effort still dedicated to taking advantage of the flexibility of NFAs to optimize device efficiencies, the implications for the stability of fullerene-free OSCs are almost completely unclear. A small number of studies have demonstrated improved lifetimes of fullerene-free OSCs under isolated environmental stress conditions (e.g., light-soaking, thermal) compared to their fullerene-based counterparts, ${ }^{6,7}$ without further evaluating their stability under mixed environmental stress factors relevant to standard operating conditions or elucidating their detailed degradation mechanisms. While the exposure to molecular oxygen and illumination has been identified as a critical environmental stress factor for fullerene-based OSCs, ${ }^{8,9}$ its impact upon fullerene-free OSCs remains unclear and has not been addressed to date. It has previously been established that two main pathways for oxygen-induced degradation exist in fullerene-based OSCs: through singlet oxygen $\left({ }^{1} \mathrm{O}_{2}\right)$ generation via energy transfer from triplet excited states and superoxide $\left(\mathrm{O}_{2}^{-}\right)$generation via photoinduced transfer of electrons from the fullerene to molecular oxygen. ${ }^{10-12} \mathrm{We}$ have demonstrated that for polymer:PCBM blends, degradation is dominated by the pathway of triplet-induced singlet oxygen generation through either the polymer ${ }^{13}$ or the fullerene $^{14}$ component, while the pathway of superoxide formation is suppressed due to a deep-lying LUMO level of PCBM. In particular, we have further demonstrated that the degradation of fullerenes, primarily through a light-induced oxidation process, can have a drastic impact upon the stability of benchmark polymer:fullerene OSCs by significantly altering the electron transport and recombination kinetics. ${ }^{8}$ However, the relevance of these degradation mechanisms for fullerenefree OSCs remains unknown, and strategies on mitigation of these potential mechanisms have not been taken into account in the development of NFAs, which offer a higher synthetic and energetic flexibility than fullerenes. The development of robust design rules for the long-term stability of fullerene-free OSCs will enable their simultaneous advances in stability and performance.

Herein, we investigate the light-induced degradation of a range of polymer:fullerene and polymer:nonfullerene OSC systems (see Figure S1 for chemical structures and full chemical names for all of the materials investigated) and found that their stability under light and oxygen is strongly correlated to the energy of the lowest unoccupied molecular orbital (LUMO) levels of the electron acceptors used. We present direct evidence linking this correlation to a fast (within $\sim 10 \mathrm{~s}$ of minutes of degradation) photo-oxidation process of the photoactive materials (including both the donor and acceptor materials), mediated by the formation of superoxide radical ions with molecular oxygen in the environment through the electron acceptors. These results unravel the critical role of energetics of the electron acceptors in environmental stability, in addition to device efficiency, of both fullerene-based and fullerene-free OSCs. Our findings highlight the importance of taking stability into account in the material design of fullerene and nonfullerene acceptors and provide a promising route in the development of high-performance and environmentally stable OSCs.

The environmental stability of a benchmark fullerene-free OSC comprising P3HT:O-IDTBR was compared to that of P3HT:PCBM under exposure to light and air. P3HT:OIDTBR represents one of the most promising fullerene-free OSC systems for commercialization to date, combining potential advantages such as low cost, good upscalability, and "burn-in" free photostability in an inert atmosphere, with largearea, industry-compatible solar modules already demonstrated. ${ }^{15}$ For the device stability studies presented herein, exposure prior to back contact deposition was performed to allow the experiments to focus on the underlying photochemical degradation of the active layer, excluding any effects of oxygen diffusion. It has previously been shown that the degradation of complete devices capped with an oxygen blocking top contact takes much longer than that of uncapped devices due to oxygen diffusion limitations. ${ }^{16}$ It is obvious that P3HT:O-IDTBR devices undergo rapid, drastic degradation, losing over $80 \%$ of their initial performance upon only $10 \mathrm{~min}$ of photoaging time, further increased to $\sim 95 \%$ loss after 30 min of exposure (Figure 1a). In comparison, P3HT:PCBM devices undergo significantly less degradation, still retaining $\sim 70 \%$ of their initial device performance upon $30 \mathrm{~min}$ of exposure (Figure $1 \mathrm{~b}$ ). The device characteristics of both OSC systems during degradation are summarized in Tables $\mathrm{S} 1$ and S2. Because the devices were degraded prior to electrode deposition, molecular oxygen was removed from the systems, and the degradation observed is due to photo-oxidation of the photoactive layers rather than oxygen acting as an electron trap. The $J-V$ characteristics of P3HT:O-IDTBR devices under dark storage in air or under exposure to AM1.5G conditions in nitrogen for $10 \mathrm{~min}$ were also measured as control experiments (Figure S2, Table S3), both revealing negligible changes in device efficiency, indicating that the rapid degradation of device efficiency in Figure 1a is primarily due to the combined exposure of light and oxygen, rather than light exposure or oxygen exposure alone. 

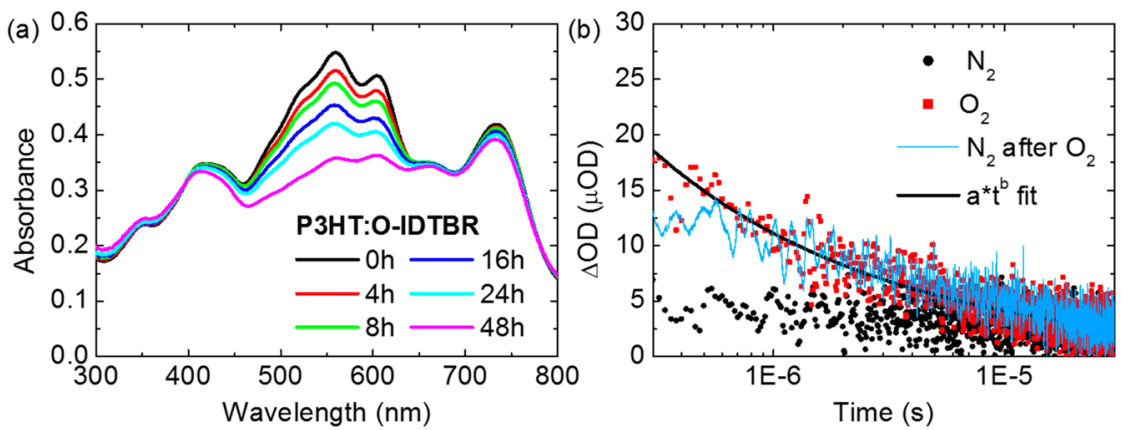

Figure 2. (a) UV-visible absorbance spectra of P3HT:O-IDTBR blend thin films photoaged in dry air for up to $48 \mathrm{~h}$. (b) Transient absorption kinetics of the P3HT:O-IDTBR blend film recorded under nitrogen $\left(\mathrm{N}_{2}\right)$, oxygen $\left(\mathrm{O}_{2}\right)$, and again $\mathrm{N}_{2}$ atmospheres (excited at 500 $\mathrm{nm}$ and probed at $1060 \mathrm{~nm}$ ). Black lines correspond to a fit to the data with a power law function, producing an exponent of -0.43 for $\mathrm{N}_{2}$ decay, revealing the polaron's reactivity with $\mathrm{O}_{2}$.
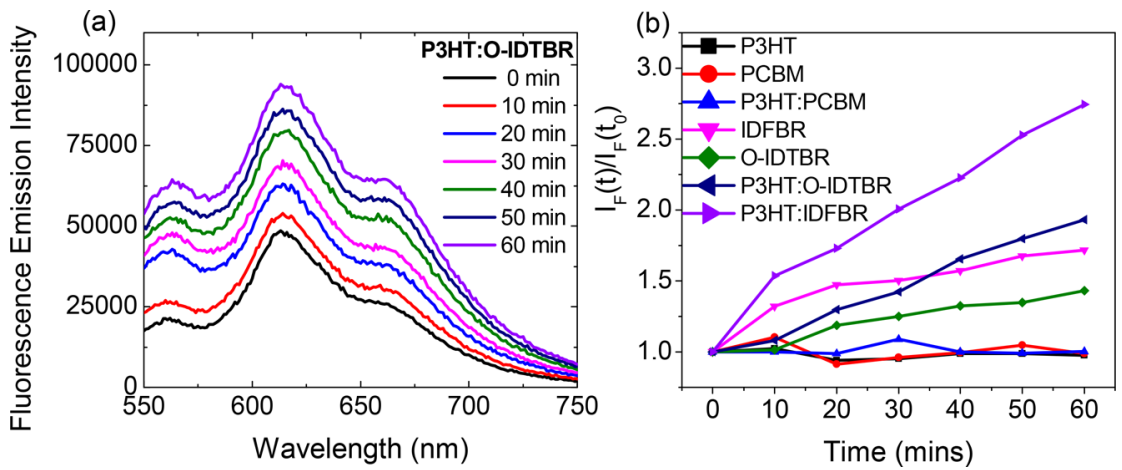

Figure 3. (a) Fluorescence spectra of the HE probe in the presence of a P3HT:O-IDTBR blend film as a function of illumination time and (b) normalized fluorescence intensity increase of the HE probe in the presence of neat and blend films at $610 \mathrm{~nm}(\mathrm{excited}$ at $520 \mathrm{~nm})$ as a function of illumination time under AM1.5G illumination conditions in dry air $(\mathrm{RH}<40 \%)$. $\operatorname{IF}(t)$ is the fluorescence maximum at time $t$, while $\operatorname{IF}\left(t_{0}\right)$ is the background fluorescence intensity. $\operatorname{IF}(t) / \operatorname{IF}\left(t_{0}\right)$ corresponds to the yield of superoxide generation.

To investigate the origin of the rapid degradation of P3HT:O-IDTBR devices, UV-visible, photoluminescence (PL), atomic force microscopy (AFM), and transient absorption spectroscopy (TAS) measurements of the blend thin films were performed. As shown in Figure 2a, P3HT:OIDTBR films undergo rapid photobleaching under white illumination in dry air, with a loss of the absorbance peak at $\sim 550 \mathrm{~nm}$ indicating the degradation of P3HT, whereas OIDTBR undergoes only modest degradation. In comparison, minimal degradation of both P3HT and O-IDTBR is seen in the UV-visible absorbance spectra of P3HT:O-IDTBR blend thin films under light exposure in nitrogen or under dark storage in air (Figure S3). PL and AFM measurements have been widely employed to characterize the bulk and surface nanomorphology of OSCs and thin films. ${ }^{17-19} \mathrm{PL}$ spectra and AFM images of the P3HT:O-IDTBR blend films (Figures S4 and S5b) reveal negligible morphological changes upon dark storage in air or photoaging in air. It thus appears that the degradation of P3HT:O-IDTBR films and devices is primarily caused by the chemical degradation of $\mathrm{P} 3 \mathrm{HT}$ due to the combined exposure of light and oxygen, with O-IDTBR showing less degradation than $\mathrm{P} 3 \mathrm{HT}$, although with some variations depending on the experimental details. In comparison, P3HT:PCBM films exhibit significantly less photobleaching upon photoaging for the same duration (Figure S6a), where the rapid degradation of P3HT compared to the acceptor was also absent. To further investigate the generality of this effect, we performed photobleaching studies for two other benchmark donor polymer systems, namely, PCDTBT and PBDB-T (Figures S7 and S8), with the results in excellent agreement with the photobleaching kinetics of P3HT-based blend films (Figure S6). We further note that the photobleaching of the blend films occurs at a greater time scale than that of the devices, commensurate with previous studies. ${ }^{8,12-14}$ The different time scale can be understood by the degradation of completed solar cells being typically much more sensitive to materials degradation, the magnitude of which, however, may be too small to be detectable by optical measurements. Figure $2 \mathrm{~b}$ shows the polaron absorption decay of a P3HT:O-IDTBR blend film recorded under dry nitrogen and dry oxygen environments. The signal assignment is based on successful fitting of the $\mathrm{N}_{2}$ decay to a power law function $(\Delta \mathrm{OD}=$ $\left.A t^{-n}\right)^{20,21}$ that is characteristic of trap-assisted bimolecular recombination of photogenerated polarons, which in chemical terms indicates photogeneration of radical ions in the film. The observed quenching by $\mathrm{O}_{2}$ therefore suggests radical ion reactivity with ambient $\mathrm{O}_{2}$. Such oxygen reactions are reported for polymer:fullerene blends with acceptors with a LUMO energy higher than that of $\mathrm{PCBM}^{12}$ as is the case for OIDTBR, and are typically assigned to generation of superoxide, $\mathrm{O}_{2}{ }^{-}$, via electron transfer from the photogenerated fullerene radical ions to $\mathrm{O}_{2}$. The data therefore suggests that the P3HT:O-IDTBR blend is sensitive to molecular oxygen and likely forms $\mathrm{O}_{2}^{-}$via electron transfer from photogenerated $\mathrm{O}$ IDTBR radical ions, further confirmed by the data in Figure 3 above. In comparison to P3HT:O-IDTBR, the transient absorption signal of the neat O-IDTBR film (Figure S9a) shows single-exponential decay with a time constant of $0.33 \mu \mathrm{s}$ 
under an inert nitrogen environment, which is consistent with triplet exciton absorption, further consistent with the degradation of O-IDTBR neat films upon photoaging (Figure 2a). The observed quenching of the triplet signal (on the 100 ns time scale) can be understood in terms of energy transfer from O-IDTBR's triplet to $\mathrm{O}_{2}$ to generate singlet oxygen. Figure $S 9 b, c$ presents the results from the transient absorption study of IDFBR and P3HT:IDFBR films, which similarly indicate mechanisms of singlet oxygen and superoxide generation identical to those found for O-IDTBR and P3HT:O-IDTBR. The P3HT:IDFBR blend film undergoes degradation during TAS measurements under an $\mathrm{O}_{2}$ environment, qualitatively agreeing with its much higher instability compared to that of the P3HT:O-IDTBR blend. Figure S10 shows the result of power law and stretched exponential fits to P3HT:O-IDTBR and P3HT:IDFBR transient absorption decays (from Figure 2), confirming their power law behavior.

To investigate the formation of superoxide, fluorescent molecular probe studies were performed, as previously reported. ${ }^{22,24}$ Hydroethidine (HE) was employed as the molecular probe due to its selective reactivity with superoxide ions to form ethidium, a fluorescent compound $\left(\lambda_{\text {excitation }}=520\right.$ $\mathrm{nm}$ and $\left.\lambda_{\text {emission }}=610 \mathrm{~nm}\right)$ insensitive to singlet oxygen, hydroxyl radicals, $\mathrm{H}_{2} \mathrm{O}_{2}$, or nitrogen radicals. ${ }^{23-25}$ Moreover, at a given illumination time, it provides a measure of the amount of superoxide generated by the sample. In particular, this probe has been recently used to study superoxide generation in hybrid perovskites, ${ }^{22}$ but here we use it to track $\mathrm{O}_{2}^{-}$generation in semiconducting polymers and small molecules. Figure $3 \mathrm{a}$ shows typical fluorescence spectra of the molecular probe for P3HT:O-IDTBR as a function of illumination time, consistent with the spectra previously measured. ${ }^{23-25}$ The increase in the fluorescence signal with longer photoaging time provides direct evidence for an increasing yield of superoxide formation as a function of illumination time. We further extend our studies to various neat and blend films of P3HT and/or fullerene/ nonfullerene acceptors, with a master plot of evolution of the superoxide formation shown in Figure $3 \mathrm{~b}$. It is obvious that the amount of superoxide formation is strongly dependent on the types of acceptors used, with O-IDTBR- and IDFBR-based neat and blend films showing the highest yield of superoxide formation and PCBM-based films showing the lowest. The blending of the acceptors with P3HT also has a strong impact, with, for example, P3HT:IDFBR film producing the highest amount of superoxide, 1.6 times more than that of the neat IDFBR film (2.74 and 1.72, respectively). The P3HT:OIDTBR film generates 1.4 times more superoxide than the neat O-IDTBR film (1.93 and 1.43, respectively). These results correlate well with the TAS data, which shows that the blend films have a greater yield of longer-lived polarons that can react with oxygen to form the superoxide. In contrast, P3HT, PCBM, and P3HT:PCBM films did not enhance the fluorescence of the molecules, implying no significant formation of superoxide, in agreement with previous studies. ${ }^{13}$

To investigate the role of superoxide in the degradation of the photoactive materials, photobleaching measurements (measured by a loss of absorbance upon degradation) were performed. Figure 4 shows the evolution of P3HT photobleaching (measured by a loss of the $\mathrm{P} 3 \mathrm{HT}$ absorbance in the blend film) in blends with various types of fullerene and nonfullerene acceptors, revealing a strong dependence of the photobleaching of $\mathrm{P} 3 \mathrm{HT}$ on the blending acceptors. It is obvious that $\mathrm{P} 3 \mathrm{HT}$ shows minimal photobleaching in blends

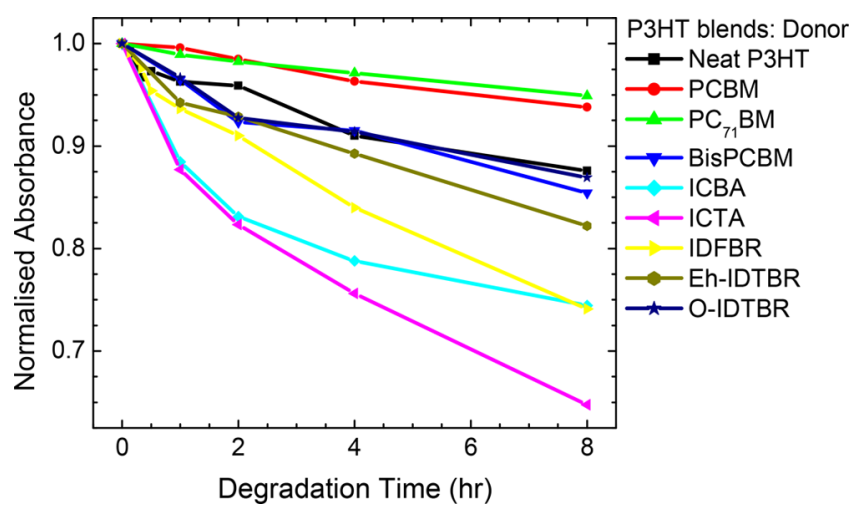

Figure 4. Evolution of the normalized P3HT absorbance peaks (see Table S4 for the wavelength at which normalization was performed for different blend systems) in blend films with different electron acceptors under AM1.5G illumination in dry air $(\mathrm{RH}<40 \%)$. The evolution of the P3HT absorbance normalized at the same wavelength of $522 \mathrm{~nm}$ and of the electron acceptor absorption peaks is also plotted as comparison in Figures S11 and S12, respectively.

with $\mathrm{PCBM}$ and $\mathrm{PC}_{71} \mathrm{BM}$ while showing more pronounced photobleaching in blends with other fullerene acceptors such as bis-PCBM, ICBA, ICTA, and NFAs such as IDFBR, OIDTBR, and EH-IDTBR. It thus appears that the superoxide formed upon photoaging can cause degradation of $\mathrm{P} 3 \mathrm{HT}$, with the severity of degradation consistent with the measured quantity of superoxide in Figure 3. The superoxide formed in the blend film can also cause material degradation of the electron acceptors (Figure S6), albeit with a greater variation than that of P3HT, likely due to the dependence of their photobleaching upon their molecular stability.

The fluorescent molecular probe and photobleaching measurements reveal a strong acceptor dependence of the stability of the photoactive materials in the blend films, whose degradation is found to be linked with superoxide formation. To quantify this strong dependence on the blending acceptors, we plot the amount of photobleaching of both P3HT and acceptors in the blend films upon $8 \mathrm{~h}$ of exposure under AM1.5G illumination conditions in dry air as a function of the LUMO levels of the neat acceptors (measured with cyclic voltammetry; see Figure S13 for the full data set). It is shown in Figure 5a that the photobleaching of $\mathrm{P} 3 \mathrm{HT}$ in the blend films correlates very well with the LUMO of the fullerenes and NFAs, with shallower LUMO levels of the acceptors resulting in more photobleaching of $\mathrm{P} 3 \mathrm{HT}$ in the blend films. The data can be fitted with an exponential growth function due to the Boltzmann dependence of the electron transfer to the molecular oxygen ground state being dependent on the LUMO energy of the acceptor. The correlation between the photobleaching of P3HT in the blend films and the LUMO levels of the acceptors also matches excellently with the fluorescence spectra (Figure 3) and is fully supported by the transient absorption and device stability measurements, confirming that the degradation of P3HT in the blend is primarily controlled by the LUMO level of the acceptors. The superoxide formed also has an analogous impact on the acceptors themselves, with a raised acceptor LUMO level generally leading to more degradation (Figure S14). On the basis of these findings, we propose a common photodegradation mechanism of OSC blend films mediated by the LUMO energy levels of electron acceptors (Figure 5b). A 
(a)

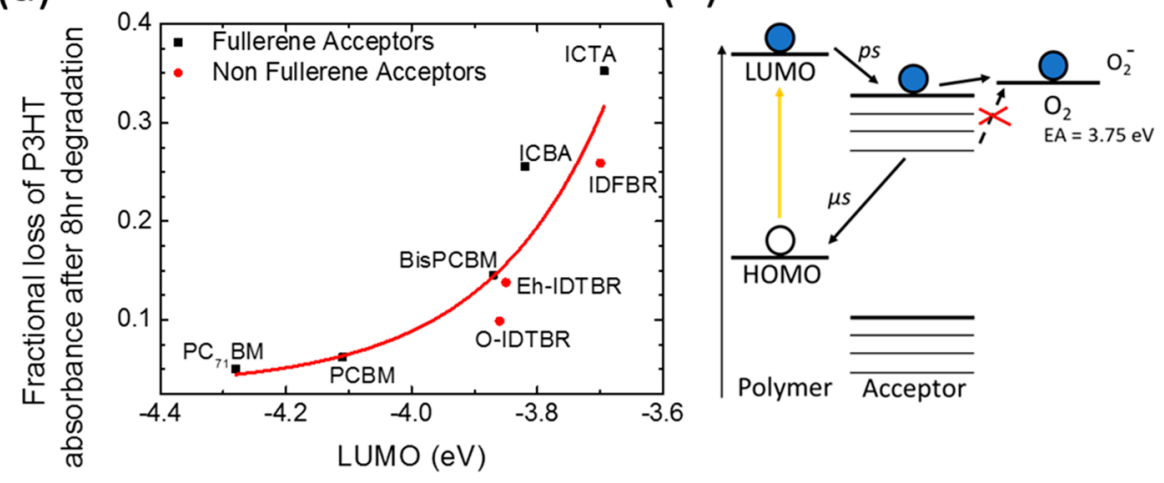

Figure 5. (a) Fractional losses of the P3HT absorbance peaks in blend films after $8 \mathrm{~h}$ of exposure under AM1.5G illumination in dry air (RH $<40 \%)$ as a function of the measured LUMO level of the acceptors, fitted with exponential growth function $y=y_{0}+A e^{\left(\left(x-x_{0}\right) / t\right)}($ red line $)$ and (b) proposed degradation mechanism, namely, the photodegradation of P3HT caused by the formation of superoxide $\left(\mathrm{O}_{2}^{-}\right)$via electron transfer from the LUMO levels of the acceptors to molecular oxygen $\left(\mathrm{O}_{2}\right)$, which has an electron affinity (EA) of $3.75 \mathrm{eV}$.

shallow LUMO level of the acceptor facilitates the transfer of electrons to molecular oxygen to form superoxide, which in turn reacts with both the electron donors and acceptors, evidenced by strong photobleaching in the blend films (e.g., P3HT:IDFBR). For electron acceptors with deeper LUMO levels, this process is energetically less favorable and is therefore suppressed (e.g., P3HT:PCBM). This process appears to be general and is independent of whether fullerene acceptors or NFAs are used. In addition, electrons at the LUMO level of the acceptor can also originate from direct photoexcitation of the acceptor and is more likely to occur in nonfullerene blend films due to their typically stronger optical absorption than that of fullerene acceptors. Furthermore, we note that the degradation of the acceptors shows greater variations than that of the $\mathrm{P} 3 \mathrm{HT}$, suggesting that their degradation may be influenced by additional factors. For example, we have reported elsewhere that the degradation of O-IDTBR and IDFBR is also strongly dependent on their molecular conformation, in addition to their LUMO levels.

Unravelling the degradation mechanisms under various environmental stress factors, in particular, under light and oxygen exposure, is fundamental to further advancing the material design of OSCs to achieve long-term environmental stability. Concerning photochemical degradation, Hoke et al. proposed a light- and oxygen-induced degradation mechanism of fullerene-based OSCs due to the photo-oxidation of polymers caused by fullerene-mediated superoxide formation. ${ }^{12}$ However, mitigation strategies of this degradation mechanism have not been taken into account in the energetic design of NFAs, with the majority of high-performance NFAs (e.g., ITIC, IDTBR) developed to date possessing a shallower LUMO level than PCBM and $\mathrm{PC}_{71} \mathrm{BM}$. Herein, we report an in-depth analysis of the degradation kinetics of benchmark polymer:fullerene and polymer:nonfullerene blend systems, including photobleaching and cyclic voltammetry measurements for a broad range of fullerene and nonfullerene acceptors, as well as a range of advanced optical, chemical, and device stability studies for selected representative systems. We present direct, quantitative evidence to demonstrate that a raised LUMO level of the acceptors above the threshold for molecular superoxide formation will lead to reduced photochemical stability of OSCs by facilitating a fast photo-oxidation process (on the minute time scale) of not only the polymer donor but also the acceptor itself. We further extend the generality of our conclusion to fullerene-free OSCs, whose degradation mechanisms under light and oxygen exposure conditions, to the best of our knowledge, have not been previously addressed.

A widely held hypothesis in the material and device design of OSCs is that individually stable photoactive materials are a good indication of the environmental stability of complete OSCs. ${ }^{26}$ For example, it is expected that P3HT:O-IDTBR blends should possess good environmental stability because both neat P3HT and O-IDTBR show fair material stability under exposure to light and oxygen (evidenced by the modest photobleaching of neat P3HT, as shown in Figure S15, and mass spectrometry data of neat IDTBR in Figure S16). Here we demonstrate that good photochemical stability of individual photoactive materials is not necessarily a good guideline for superior stability of OSCs under the same degradation conditions because the blending of individual materials could turn on additional critical degradation pathways. For polymer:PCBM blends, the light- and oxygen-induced degradation is primarily driven by singlet oxygen generation from polymer triplet states, with this pathway being suppressed in P3HT:PCBM blends due to the short triplet lifetime of regioregular $\mathrm{P} 3 \mathrm{HT}$ and the relatively slow degradation of P3HT:PCBM blends observed herein most likely originating from PCBM triplet-induced singlet oxygen generation. ${ }^{8,13,14}$ When the LUMO level of the acceptor is raised, however, a different degradation pathway based on superoxide generation turns on. This is of particular relevance to polymer:nonfullerene OSCs due to ease of tunability of the energetics of NFAs. This degradation pathway is less critical for conventional fullerene-based OSCs, whereby the formation of superoxide is suppressed due to a deeper-lying LUMO energy level of $\mathrm{PCBM}$ or $\mathrm{PC}_{71} \mathrm{BM}$.

It should also be noted that currently the most widely used electron donors of fullerene-free OSCs are still those originally optimized for fullerenes, e.g., poly(3-hexylthiophene) (P3HT), PTB7-Th (PCE10), PffBT4T-2OD (PCE11), and PBDB-T (PCE12), with most high-performance NFAs developed to date optimized to match these (or energetically similar) donor polymers. ${ }^{1,6,27,28}$ One such optimization strategy has been designing NFAs with a raised LUMO level in order to achieve a higher $V_{\text {oc }}$ (and hence power conversion efficiency) by creating a higher donor HOMO/acceptor LUMO offset. We have demonstrated that such a strategy may lead to a 
significant decrease in the photochemical stability of the resulting OSCs by significantly increasing the yield of superoxide formation. Our results highlight the complexity in the material design to simultaneously achieve superior efficiency and stability of OSCs. A redesign of the NFAs with deeper LUMO energy levels, as well as their matching donor polymers with deepened HOMO levels to compensate the $V_{\text {oc }}$ loss, might be a promising route toward the development of both efficient and environmentally stable fullerene-free OSCs.

In conclusion, we present a range of advanced optical, chemical, and device stability measurements to investigate the degradation mechanisms of benchmark fullerene-based and fullerene-free OSCs. We establish the critical correlation between the LUMO level of the fullerene and nonfullerene electron acceptors and the resulting environmental stability of OSCs and blend films strongly mediated by the yield of superoxide formation. Our results highlight the need to redesign electron acceptor materials with deepened LUMO energy levels, as well as their donor polymers with deepened HOMO levels, to overcome the challenge of simultaneously improving device efficiency and stability, thereby paving the way toward simultaneously achieving high efficiency and longterm environmental stability of OSCs.

\section{ASSOCIATED CONTENT}

\section{S Supporting Information}

The Supporting Information is available free of charge on the ACS Publications website at DOI: 10.1021/acsenergylett.9b00109.

Experimental details and additional experimental data, including chemical structures, device characteristics, current density-voltage characteristics, UV-visible absorbance spectra, photoluminescence spectra, AFM images, transient absorption decays, power and exponential law fits, absorbance peaks, CV scans, fractional losses/changes of absorbance peaks, and negative ion mode MALDI-TOF measurements (PDF)

\section{AUTHOR INFORMATION}

\section{Corresponding Authors}

*E-mail: w.c.tsoi@swansea.ac.uk.

*E-mail: liz75@cardiff.ac.uk.

\section{ORCID}

Joel Luke: 0000-0003-3208-8572

Andrew Wadsworth: 0000-0002-9050-0599

Iain McCulloch: 0000-0002-6340-7217

Ji-Seon Kim: 0000-0003-4715-3656

Saif A. Haque: 0000-0001-5483-3321

James R. Durrant: 0000-0001-8353-7345

Stoichko D. Dimitrov: 0000-0002-1564-7080

Wing C. Tsoi: 0000-0003-3836-5139

Zhe Li: 0000-0003-1149-7000

\section{Notes}

The authors declare no competing financial interest.

\section{ACKNOWLEDGMENTS}

H.K.H.L., W.C.T., and J.D. thank the Welsh Assembly Government of the Ser Cymru Solar Program, and Z.L. and S.D. thank the Welsh Assembly Government Ser Cymru II fellowship scheme for financial support. E.M.S. and W.C.T. thank the National Research Network in Advanced Engineering and Materials and EPSRC funded Project EP/M025020/1. This work is partially funded by the European regional Development Fund through the Welsh Government. A.J.C. and W.C.T. acknowledge funding from the European Social Fund via the Welsh Government and EPSRC Project EP/ L015099/1. J.L. and J.S.K. acknowledge the UK EPSRC for the Plastic Electronics Centre for Doctoral Training (EP/ L016702/1) funding and CSEM Brasil for studentship.

\section{REFERENCES}

(1) Baran, D.; Ashraf, R. S.; Hanifi, D. A.; Abdelsamie, M.; Gasparini, N.; Röhr, J. A.; Holliday, S.; Wadsworth, A.; Lockett, S.; Neophytou, M.; et al. Reducing the Efficiency-Stability-Cost Gap of Organic Photovoltaics with Highly Efficient and Stable Small Molecule Acceptor Ternary Solar Cells. Nat. Mater. 2017, 16, 363.

(2) Meng, L.; Zhang, Y.; Wan, X.; Li, C.; Zhang, X.; Wang, Y.; Ke, X.; Xiao, Z.; Ding, L.; Xia, R.; et al. Organic and Solution-Processed Tandem Solar Cells with 17.3\% Efficiency. Science 2018, 361, 10941098.

(3) Li, S.; Ye, L.; Zhao, W.; Yan, H.; Yang, B.; Liu, D.; Li, W.; Ade, H.; Hou, J. A Wide Band Gap Polymer with a Deep Highest Occupied Molecular Orbital Level Enables 14.2\% Efficiency in Polymer Solar Cells. J. Am. Chem. Soc. 2018, 140, 7159-7167.

(4) Baran, D.; Kirchartz, T.; Wheeler, S.; Dimitrov, S.; Abdelsamie, M.; Gorman, J.; Ashraf, R. S.; Holliday, S.; Wadsworth, A.; Gasparini, N.; et al. Reduced Voltage Losses Yield 10\% Efficient Fullerene Free Organic Solar Cells with > 1 V Open Circuit Voltages. Energy Environ. Sci. 2016, 9, 3783-3793.

(5) Qian, D.; Zheng, Z.; Yao, H.; Tress, W.; Hopper, T. R.; Chen, S.; Li, S.; Liu, J.; Chen, S.; Zhang, J.; et al. Design Rules for Minimizing Voltage Losses in High-Efficiency Organic Solar Cells. Nat. Mater. 2018, 17, 703-709.

(6) Cha, H.; Wu, J.; Wadsworth, A.; Nagitta, J.; Limbu, S.; Pont, S.; Li, Z.; Searle, J.; Wyatt, M. F.; Baran, D.; et al. An Efficient, "Burn in" Free Organic Solar Cell Employing a Nonfullerene Electron Acceptor. Adv. Mater. 2017, 29, 1701156.

(7) Gasparini, N.; Salvador, M.; Strohm, S.; Heumueller, T.; Levchuk, I.; Wadsworth, A.; Bannock, J. H.; de Mello, J. C.; Egelhaaf, H.-J.; Baran, D.; et al. Burn-in Free Nonfullerene-Based Organic Solar Cells. Adv. Energy Mater. 2017, 7, 1700770.

(8) Lee, H. K. H.; Telford, A. M.; Röhr, J. A.; Wyatt, M. F.; Rice, B.; Wu, J.; de Castro Maciel, A.; Tuladhar, S. M.; Speller, E.; McGettrick, J.; et al. The Role of Fullerenes in the Environmental Stability of Polymer:Fullerene Solar Cells. Energy Environ. Sci. 2018, 11, 417428.

(9) Wang, Y.; Jafari, M. J.; Wang, N.; Qian, D.; Zhang, F.; Ederth, T.; Moons, E.; Wang, J.; Inganäs, O.; Huang, W.; et al. Light-Induced Degradation of Fullerenes in Organic Solar Cells: a Case Study on TQ1:PC ${ }_{71}$ BM. J. Mater. Chem. A 2018, 6, 11884-11889.

(10) Fraga Domínguez, I.; Distler, A.; Lüer, L. Stability of Organic Solar Cells: The Influence of Nanostructured Carbon Materials. Adv. Energy Mater. 2017, 7, 1601320.

(11) Distler, A.; Kutka, P.; Sauermann, T.; Egelhaaf, H.-J.; Guldi, D. M.; Di Nuzzo, D.; Meskers, S. C. J.; Janssen, R. A. J. Effect of PCBM on the Photodegradation Kinetics of Polymers for Organic Photovoltaics. Chem. Mater. 2012, 24, 4397-4405.

(12) Hoke, E. T.; Sachs-Quintana, I. T.; Lloyd, M. T.; Kauvar, I.; Mateker, W. R.; Nardes, A. M.; Peters, C. H.; Kopidakis, N.; McGehee, M. D. The Role of Electron Affinity in Determining Whether Fullerenes Catalyze or Inhibit Photooxidation of Polymers for Solar Cells. Adv. Energy Mater. 2012, 2, 1351-1357.

(13) Soon, Y. W.; Shoaee, S.; Ashraf, R. S.; Bronstein, H.; Schroeder, B. C.; Zhang, W.; Fei, Z.; Heeney, M.; McCulloch, I.; Durrant, J. R. Material Crystallinity as a Determinant of Triplet Dynamics and Oxygen Quenching in Donor Polymers for Organic Photovoltaic Devices. Adv. Funct. Mater. 2014, 24, 1474-1482. 
(14) Speller, E. M.; McGettrick, J. D.; Rice, B.; Telford, A. M.; Lee, H. K. H.; Tan, C.-H.; De Castro, C. S.; Davies, M. L.; Watson, T. M.; Nelson, J.; et al. Impact of Aggregation on the Photochemistry of Fullerene Films: Correlating Stability to Triplet Exciton Kinetics. ACS Appl. Mater. Interfaces 2017, 9, 22739-22747.

(15) Strohm, S.; Machui, F.; Langner, S.; Kubis, P.; Gasparini, N.; Salvador, M.; McCulloch, I.; Egelhaaf, H. J.; Brabec, C. J. P3HT: Non-Fullerene Acceptor Based Large Area, Semi-Transparent PV Modules with Power Conversion Efficiencies of 5\%, Processed by Industrially Scalable Methods. Energy Environ. Sci. 2018, 11 (8), $2225-2234$.

(16) Shoaee, S.; Durrant, J. R. Oxygen Diffusion Dynamics in Organic Semiconductor Films. J. Mater. Chem. C 2015, 3, 1007910084 .

(17) Drees, M.; Hoppe, H.; Winder, C.; Neugebauer, H.; Sariciftci, N. S.; Schwinger, W.; Schaffler, F.; Topf, C.; Scharber, M. C.; Zhu, Z.; et al. Stabilization of the nanomorphology of polymer-fullerene "bulk heterojunction" blends using a novel polymerizable fullerene derivative. J. Mater. Chem. 2005, 15, 5158-5163.

(18) Utzat, H.; Dimitrov, S. D.; Wheeler, S.; Collado-Fregoso, E.; Tuladhar, P. S.; Schroeder, B. C.; McCulloch, I.; Durrant, J. R. Charge Separation in Intermixed Polymer: $\mathrm{PC}_{70} \mathrm{BM}$ Photovoltaic Blends: Correlating Structural and Photophysical Length Scales as a Function of Blend Composition. J. Phys. Chem. C 2017, 121, 9790-9801.

(19) Wong, H. C.; Li, Z.; Tan, C. H.; Zhong, H.; Huang, Z.; Bronstein, H.; McCulloch, I.; Cabral, J. T.; Durrant, J. R. Morphological Stability and Performance of Polymer-Fullerene Solar Cells under Thermal Stress: The Impact of Photoinduced $\mathrm{PC}_{60} \mathrm{BM}$ Oligomerization. ACS Nano 2014, 8, 1297-1308.

(20) Shuttle, C. G.; O’Regan, B.; Ballantyne, A. M.; Nelson, J.; Bradley, D. D. C.; Durrant, J. R. Bimolecular Recombination Losses in Polythiophene: Fullerene Solar Cells. Phys. Rev. B: Condens. Matter Mater. Phys. 2008, 78, 113201.

(21) Nelson, J. Diffusion-Limited Recombination in PolymerFullerene Blends and Its Influence on Photocurrent Collection. Phys. Rev. B: Condens. Matter Mater. Phys. 2003, 67, 155209.

(22) Aristidou, N.; Eames, C.; Sanchez-Molina, I.; Bu, X.; Kosco, J.; Islam, M. S.; Haque, S. A. Fast Oxygen Diffusion and Iodide Defects Mediate Oxygen-Induced Degradation of Perovskite Solar Cells. Nat. Commun. 2017, 8, 15218.

(23) Gomes, A.; Fernandes, E.; Lima, J. L. F. C. Fluorescence Probes Used for Detection of Reactive Oxygen Species. J. Biochem. Biophys. Methods 2005, 65, 45-80.

(24) Aristidou, N.; Sanchez-Molina, I.; Chotchuangchutchaval, T.; Brown, M.; Martinez, L.; Rath, T.; Haque, S. A. The Role of Oxygen in the Degradation of Methylammonium Lead Trihalide Perovskite Photoactive Layers. Angew. Chem., Int. Ed. 2015, 54, 8208-8212.

(25) Bryant, D.; Aristidou, N.; Pont, S.; Sanchez-Molina, I.; Chotchunangatchaval, T.; Wheeler, S.; Durrant, J. R.; Haque, S. A. Light and Oxygen Induced Degradation Limits the Operational Stability of Methylammonium Lead Triiodide Perovskite Solar Cells. Energy Environ. Sci. 2016, 9, 1655-1660.

(26) Jørgensen, M.; Norrman, K.; Gevorgyan, S. A.; Tromholt, T.; Andreasen, B.; Krebs, F. C. Stability of Polymer Solar Cells. Adv. Mater. 2012, 24, 580-612.

(27) Holliday, S.; Ashraf, R. S.; Wadsworth, A.; Baran, D.; Yousaf, S. A.; Nielsen, C. B.; Tan, C.-H.; Dimitrov, S. D.; Shang, Z.; Gasparini, N.; et al. High-Efficiency and Air-Stable P3HT-Based Polymer Solar Cells with a New Non-Fullerene Acceptor. Nat. Commun. 2016, 7, 11585 .

(28) Zhao, W.; Qian, D.; Zhang, S.; Li, S.; Inganäs, O.; Gao, F.; Hou, J. Fullerene-Free Polymer Solar Cells with over 11\% Efficiency and Excellent Thermal Stability. Adv. Mater. 2016, 28, 4734-4739. 